\title{
Piecewise Constant Perturbation Methods for the Multichannel Schrödinger Equation
}

\author{
Veerle Ledoux ${ }^{\star}$, Marnix Van Daele, and Guido Vanden Berghe \\ Vakgroep Toegepaste Wiskunde en Informatica, Ghent University, \\ Krijgslaan 281-S9, B-9000 Gent, Belgium \\ \{Veerle.Ledoux, Marnix.VanDaele, Guido.VandenBerghe\}@UGent.be
}

\begin{abstract}
The $\mathrm{CPM}\{P, N\}$ methods form a class of methods specially devised for the propagation of the solution of the one-dimensional Schrödinger equation. Using these $\operatorname{CPM}\{P, N\}$ methods in a shooting procedure, eigenvalues of the boundary value problem are obtained to very high precision. Some recent advances allowed the generalization of the $\operatorname{CPM}\{P, N\}$ methods to systems of coupled Schrödinger equations. Also for these generalised $\mathrm{CPM}\{P, N\}$ methods a shooting procedure can be formulated, solving the multichannel bound state problem.
\end{abstract}

\section{Introduction}

There are many problems in quantum chemistry, theoretical physics, atomic and molecular physics, and physical chemistry that can be transformed into the solution of coupled differential equations of Schrödinger type. Such a system of coupled equations may be written in matrix notation

$$
\frac{d^{2} \mathbf{y}(x)}{d x^{2}}=[\mathbf{V}(x)-E \mathbf{I}] \mathbf{y}(x) .
$$

If there are $n$ channels, $\mathbf{y}(x)$ is a column vector of order $n, \mathbf{I}$ is the $n \times n$ unity matrix. The potential energy $n \times n$ matrix $\mathbf{V}(x)$ will be assumed throughout the following to be symmetric, which is often the case in molecular scattering and bound state applications.

There are various approaches to the solution of the coupled equations (11) (see a.o. 1, 2, 5, 14, 15]). In the more early work approximate schemes were used which attempt to reduce the coupled equations to a set of one-dimensional problems (e.g. in 14). A more modern approach is to propagate the solutions numerically, without reducing them to one-dimensional form. A large number of numerical methods have been suggested for carrying out the propagation. However when bound state boundary conditions are applied, acceptable solutions of the coupled equations exist only when $E$ is an eigenvalue of the Hamiltonian and additional techniques are needed to locate the eigenvalues. Early methods for doing this were developed by Gordon 4 and Johnson [10.

\footnotetext{
^ Research Assistant of the Fund for Scientific Research - Flanders (Belgium) (F.W.O.Vlaanderen).
} 
Recently it has been shown in 12 that higher order Piecewise Constant Perturbation ( $\mathrm{CP}$ ) methods can be constructed for the propagation of the solution of Eq. (1). Here we will show how such a CP method can be used in a shooting procedure to solve the associated eigenvalue problem, that is to estimate the values of $E$ for which a solution of Eq. (1) exists satisfying some boundary conditions in the endpoints of the integration interval.

\section{The CP Algorithm for the Multichannel Case}

The Piecewise Perturbation methods (PPM) have been successfully applied for the propagation of the solution of a one-dimensional Schrödinger problem (see 6, 7]). The PPM idea is to replace (piecewisely) the given equation by another differential equation, called the reference differential equation, which can be solved exactly. The deviation of the solution of the reference equation from the solution of the original equation is further estimated by means of the perturbation theory.

The CP methods form a subclass of the PPM where the potential function $V$ of the reference equation is a piecewise constant. In [7] and [1] some higher order $\mathrm{CP}$ versions, the so-called $\mathrm{CPM}\{P, N\}$ methods were found to be well suited for the solution of the one-dimensional Schrödinger problem. More recently these $\operatorname{CPM}\{P, N\}$ formulae were generalised to the coupled channel case (see [12]). In this section the main elements of the $\operatorname{CPM}\{P, N\}$ algorithm are recapitulated briefly. In the following description bold type indicates a column vector or matrix.

Let $[X, X+h]$ be the current one step interval of the partition of the integration interval. The multichannel Schrödinger problem

$$
\mathbf{y}^{\prime \prime}=(\mathbf{V}(x)-E \mathbf{I}) \mathbf{y}
$$

is considered on this interval. The algorithm of the CP method links the values of the solution at the two ends of the interval in the following two ways, to be used for forwards and backwards propagation,

$$
\left[\begin{array}{c}
\mathbf{y}(X+h) \\
\mathbf{y}^{\prime}(X+h)
\end{array}\right]=\mathbf{T}^{f}(h)\left[\begin{array}{c}
\mathbf{y}(X) \\
\mathbf{y}^{\prime}(X)
\end{array}\right], \quad\left[\begin{array}{c}
\mathbf{y}(X) \\
\mathbf{y}^{\prime}(X)
\end{array}\right]=\mathbf{T}^{b}(h)\left[\begin{array}{c}
\mathbf{y}(X+h) \\
\mathbf{y}^{\prime}(X+h)
\end{array}\right]
$$

To construct $\mathbf{T}^{f}$ and $\mathbf{T}^{b}$ we use two particular solutions of the local problem

$$
\mathbf{y}^{\prime \prime}=(\mathbf{V}(X+\delta)-E \mathbf{I}) \mathbf{y}, \quad \delta \in[0, h] .
$$

Specifically, if $\mathbf{u}(\delta)$ and $\mathbf{v}(\delta)$ are the $n \times n$ solutions corresponding to the initial conditions $\mathbf{y}(0)=\mathbf{I}, \mathbf{y}^{\prime}(0)=\mathbf{0}$ and $\mathbf{y}(0)=\mathbf{0}, \mathbf{y}^{\prime}(0)=\mathbf{I}$, respectively $(\mathbf{0}$ is the $n$ by $n$ zero matrix) then $\mathbf{T}^{f}$ and $\mathbf{T}^{b}$ have the form

$$
\mathbf{T}^{f}(\delta)=\left[\begin{array}{cc}
\mathbf{u}(\delta) & \mathbf{v}(\delta) \\
\mathbf{u}^{\prime}(\delta) & \mathbf{v}^{\prime}(\delta)
\end{array}\right], \quad \mathbf{T}^{b}(\delta)=\left[\begin{array}{cc}
\mathbf{v}^{\prime}(\delta) & -\mathbf{v}(\delta) \\
-\mathbf{u}^{\prime}(\delta) & \mathbf{u}(\delta)
\end{array}\right],
$$


To determine $\mathbf{u}$ and $\mathbf{v}$ the potential matrix is approximated by a truncated series over the shifted Legendre polynomials $P_{n}^{*}(\delta / h)$. The used parametrization is

$$
\mathbf{V}(X+\delta)=\sum_{m=0}^{N} \mathbf{V}_{m} h^{m} P_{m}^{*}(\delta / h)
$$

where the matrix weights are calculated by quadrature $\left(\overline{\mathbf{V}}_{m}=\mathbf{V}_{m} h^{m+2}, m=\right.$ $1,2, \ldots)$,

$$
\begin{aligned}
\mathbf{V}_{0} & =\frac{1}{h} \int_{0}^{h} \mathbf{V}(X+\delta) d \delta, \\
\overline{\mathbf{V}}_{m} & =(2 m+1) h \int_{0}^{h} \mathbf{V}(X+\delta) P_{m}^{*}(\delta / h) d \delta, \quad m=1,2,3, \ldots .
\end{aligned}
$$

The symmetric matrix $\mathbf{V}_{0}$ is then diagonalized and let $\mathbf{D}$ be the diagonalization matrix. In the $\mathbf{D}$ representation Eq. (3) becomes

$$
\mathbf{y}^{\mathbf{D}^{\prime \prime}}=\left(\sum_{m=0}^{N} \mathbf{V}_{m}^{\mathbf{D}} h^{m} P_{m}^{*}(\delta / h)-E \mathbf{I}\right) \mathbf{y}^{\mathbf{D}}, \quad \delta \in[0, h]
$$

and this is solved for $\mathbf{u}^{\mathbf{D}}$ and $\mathbf{v}^{\mathbf{D}}$; the initial conditions are the same as in the original representation. The perturbation procedure is used, in which the diagonal matrix $\mathbf{V}_{\mathbf{0}}^{\mathrm{D}}$ is the reference potential and

$$
\Delta \mathbf{V}=\sum_{m=1}^{N} \mathbf{V}_{m}^{\mathbf{D}} h^{m} P_{m}^{*}(\delta / h)
$$

is the perturbation.

First, the matrices of functions $\mathbf{u}^{\mathbf{D}}(\delta)$ and $\mathbf{v}^{\mathbf{D}}(\delta)$, denoted generically as $\mathbf{p}(\delta)$, are written as a perturbation series:

$$
\mathbf{p}(\delta)=\mathbf{p}_{0}(\delta)+\mathbf{p}_{1}(\delta)+\mathbf{p}_{2}(\delta)+\mathbf{p}_{3}(\delta)+\ldots
$$

where the zeroth order term $\mathbf{p}_{0}(\delta)$ is the solution of

$$
\mathbf{p}_{0}^{\prime \prime}=\left(\mathbf{V}_{\mathrm{o}}^{\mathbf{D}}-E\right) \mathbf{p}_{0}
$$

with $\mathbf{p}_{0}(0)=\mathbf{I}, \mathbf{p}_{0}^{\prime}(0)=\mathbf{0}$ for $\mathbf{u}_{0}$ and $\mathbf{p}_{0}(0)=\mathbf{0}, \mathbf{p}_{0}^{\prime}(0)=\mathbf{I}$ for $\mathbf{v}_{0}$. Let the functions $\xi(Z)$ and $\eta_{0}(Z)$ be defined as follows:

$$
\xi(Z)= \begin{cases}\cos \left(|Z|^{1 / 2}\right) & \text { if } Z \leq 0, \\ \cosh \left(Z^{1 / 2}\right) & \text { if } Z>0,\end{cases}
$$

and

$$
\eta_{0}(Z)= \begin{cases}\sin \left(|Z|^{1 / 2}\right) /|Z|^{1 / 2} & \text { if } Z<0 \\ 1 & \text { if } Z=0 \\ \sinh \left(Z^{1 / 2}\right) / Z^{1 / 2} & \text { if } Z>0\end{cases}
$$


while $\eta_{s}(Z)$ with $s>0$ are further generated by recurrence :

$$
\begin{aligned}
& \eta_{1}(Z)=\left[\xi(Z)-\eta_{0}(Z)\right] / Z, \\
& \eta_{s}(Z)=\left[\eta_{s-2}(Z)-(2 s-1) \eta_{s-1}(Z)\right] / Z, \quad s=2,3, \ldots .
\end{aligned}
$$

The zeroth order propagators $\mathbf{u}_{0}(\delta)$ and $\mathbf{u}_{0}(\delta)$ are then diagonal matrices, defined as follows:

$$
\begin{gathered}
\mathbf{u}_{0}=\mathbf{v}_{0}^{\prime}=\xi(\mathbf{Z}) \\
\delta \mathbf{u}_{0}^{\prime}=\mathbf{Z}(\delta) \eta_{0}(\mathbf{Z}) \\
\mathbf{v}_{0}=\delta \eta_{0}(\mathbf{Z})
\end{gathered}
$$

where

$$
\mathbf{Z}(\delta)=\left(\mathbf{V}_{0}^{\mathbf{D}}-E \mathbf{I}\right) \delta^{2}
$$

and $\xi(\mathbf{Z}), \eta_{m}(\mathbf{Z})$ two $n \times n$ diagonal matrices of functions with $\xi\left(Z_{i}\right)$, resp. $\eta_{m}\left(Z_{i}\right)$ as $i^{t h}$ diagonal element (with $\left.Z_{i}(\delta)=\left(V_{0_{i i}}^{D}-E\right) \delta^{2}\right)$.

The $n \times n$ 'correction' matrix of functions $\mathbf{p}_{q}$ is the solution of the system

$$
\mathbf{p}_{q}^{\prime \prime}=\left(\mathbf{V}_{\mathrm{o}}^{\mathbf{D}}-E I\right) \mathbf{p}_{q}+\Delta \mathbf{V}(\delta) \mathbf{p}_{q-1}, \quad \mathbf{p}_{q}(0)=\mathbf{p}_{q}^{\prime}(0)=0 .
$$

The following iteration procedure exists to construct the corrections.

Correction $\mathbf{p}_{q-1}\left(\mathbf{p}=\mathbf{u}^{\mathbf{D}}, \mathbf{v}^{\mathbf{D}}\right)$ is assumed to be known and of such a form that the product $\Delta \mathbf{V}(\delta) \mathbf{p}_{q-1}$ reads

$$
\Delta \mathbf{V}(\delta) \mathbf{p}_{q-1}(\delta)=\mathbf{Q}(\delta) \xi(\mathbf{Z})+\sum_{m=0}^{+\infty} \delta^{2 m+1} \mathbf{R}_{m}(\delta) \eta_{m}(\mathbf{Z})
$$

Then $\mathbf{p}_{q}(\delta)$ and $\mathbf{p}_{q}^{\prime}(\delta)$ are of the form

$$
\begin{aligned}
\mathbf{p}_{q}(\delta) & =\sum_{m=0}^{+\infty} \delta^{2 m+1} \mathbf{C}_{m}(\delta) \eta_{\mathbf{m}}(\mathbf{Z}) \\
\mathbf{p}_{q}^{\prime}(\delta) & =\mathbf{C}_{0}(\delta) \xi(\mathbf{Z})+\sum_{m=0}^{+\infty} \delta^{2 m+1}\left(\frac{d \mathbf{C}_{m}(\delta)}{d \delta}+\delta \mathbf{C}_{m+1}(\delta)\right) \eta_{m}(\mathbf{Z})
\end{aligned}
$$

where all $\mathbf{C}_{m}$ matrices are given by quadrature:

$$
\begin{aligned}
\mathbf{C}_{0}(\delta) & =\frac{1}{2} \int_{0}^{\delta} \mathbf{Q}\left(\delta_{1}\right) d \delta_{1} \\
\mathbf{C}_{m}(\delta) & =\frac{1}{2} \delta^{-m} \int_{0}^{\delta} \delta_{1}^{m-1}\left(\mathbf{R}_{m-1}\left(\delta_{1}\right)-\frac{d^{2} \mathbf{C}_{m-1}\left(\delta_{1}\right)}{d \delta_{1}^{2}}-\left[\mathbf{C}_{m-1}\left(\delta_{1}\right), \mathbf{V}_{\mathrm{o}}^{\mathbf{D}}\right]\right) d \delta_{1}
\end{aligned}
$$

where $\left[\mathbf{C}_{m-1}, \mathbf{V}_{\mathrm{o}}^{\mathbf{D}}\right]$ is the commutator of the matrices $\mathbf{C}_{m-1}$ and $\mathbf{V}_{\mathrm{o}}^{\mathbf{D}}$. 
To calculate successive corrections for $\mathbf{u}^{\mathbf{D}}$, the starting functions in $\Delta \mathbf{V}(\delta) \mathbf{p}_{0}(\delta)$ are $\mathbf{Q}(\delta)=\Delta \mathbf{V}$ and $\mathbf{R}_{\mathbf{0}}(\delta)=\mathbf{R}_{1}(\delta)=\ldots=\mathbf{0}$. For $\mathbf{v}^{\mathbf{D}}$ the starting functions are $\mathbf{Q}(\delta)=\mathbf{0}, \mathbf{R}_{0}(\delta)=\Delta \mathbf{V}(\delta), \mathbf{R}_{1}(\delta)=\mathbf{R}_{2}(\delta)=\ldots=0$. We have thus all ingredients necessary to evaluate the perturbative corrections.

Once the values at $h$ of the $\mathbf{u}^{\mathbf{D}}, \mathbf{v}^{\mathbf{D}}$ matrices and of their derivatives have been evaluated, they are reconverted to the original representation to obtain the desired $\mathbf{T}^{f}$ and $\mathbf{T}^{b}$.

This theory was used to construct some CP versions which are identified as $\operatorname{CPM}\{P, N\}$ methods. Such a $\operatorname{CPM}\{P, N\}$ algorithm is of maximum order $P$ at low energies and of order $N$ in the asymptotic regime. As shown in [12] a $\operatorname{CPM}\{P, N\}$ method can be used to efficiently propagate the solution of Eq. (2) (forwards and backwards).

\section{The Boundary Value Problem}

We now consider the eigenvalue problem arising when boundary conditions are introduced at both ends of the integration interval. Values of $E$ have to be found for which a solution of (1) exists satisfying the boundary conditions.

Before proceeding to the multichannel case, it is instructive to consider the procedure used for the single-channel (one-dimensional) Schrödinger equation. In the one-dimensional case $y(x)$ and $V(x)$ are both scalar quantities

$$
y^{\prime \prime}(x)=[V(x)-E] y(x), \quad x \in(a \geq-\infty, b \leq+\infty) .
$$

In this simple case, the eigenvalue problem has been solved since the early work of Cooley [3] based on the widely used shooting method. Such a shooting method proceeds as follows: A trial value $E_{\text {trial }}$ of $E$ is chosen and the following steps are used: (i) we start at the beginpoint of the integration interval $a$ and propagate the solution (e.g. using a CP method) towards the endpoint of the integration interval $b$. We stop at an arbitrary point $x_{\text {match }}$. (ii) We then start at $b$ and we propagate backwards until $x_{\text {match }}$ is reached. (iii) At $x_{\text {match }}$ the left-hand and right-hand solutions are matched. The two solutions are arbitrarily normalised, so their values can always made to agree at the matching point by renormalising them. However, the criterion for $E_{\text {trial }}$ to be an eigenvalue is that the derivatives $y^{\prime}$ should match, as well as the values. The matching condition is thus

$$
\frac{y_{L}^{\prime}}{y_{L}}=\frac{y_{R}^{\prime}}{y_{R}},
$$

or equivalently

$$
\operatorname{det}\left(\begin{array}{ll}
y_{L} & y_{R} \\
y_{L}^{\prime} & y_{R}^{\prime}
\end{array}\right)=0,
$$

where the subscripts $L$ and $R$ indicate outwards and inwards solutions originating at $a$ and $b$ respectively. The matching function $y_{L} y_{R}^{\prime}-y_{R} y_{L}^{\prime}$ is thus a function of the energy that is zero when $E_{\text {trial }}$ is an eigenvalue. If $E_{\text {trial }}$ is not found to be an eigenvalue, steps (i)-(iii) are repeated with an adjusted value of $E_{\text {trial }}$. It is 
possible to obtain a new $E_{\text {trial }}$ value simply by using one of the standard numerical procedures for finding a zero of a function. For the CP methods a NewtonRaphson iteration procedure can be formulated, because the $\mathrm{CP}$ algorithm allows a direct evaluation of the first derivatives of the solution with respect to the energy $E$ (see [7]). The software packages SLCPM12 [8] and MATSLISE [13] use the $\mathrm{CP}$ methods in combination with this Newton-Raphson process to obtain very accurate eigenvalue estimations for the one-dimensional Schrödinger problem.

For a system of $n$ coupled equations, a procedure can be used which is largely inspired from the method outlined above for the one-dimensional problem. In the multichannel case, the desired wavefunction is a column vector $\mathbf{y}(x)$. In order to start propagating a solution to the coupled equations, it is necessary to know not only the initial values of the elements of $\mathbf{y}(x)$ at $a$ and $b$ (which are given by the boundary conditions) but also their derivatives. In the single-channel case, the initial derivatives are arbitrary, because their effects are cancelled by renormalising the function after the matching point was reached. However, in the multichannel case the situation is more complicated: here the ratios of the initial derivatives in the different channels are significant. In early methods, schemes were devised for converging upon suitable initial derivatives as well as the eigenvalue. However, Gordon [4] has devised a method that avoids the problem of searching for the correct values of the initial boundary derivatives. Instead of propagating a single wavefunction vector, a complete set of $n$ vectors is propagated, spanning the space of all possible initial derivatives. So the wavefunction becomes an $n \times n$ matrix $\mathbf{Y}(x)$ instead of a column vector $\mathbf{y}(x)$. Since the columns of $\mathbf{Y}(x)$ span the space of all possible initial derivatives, any wavefunction that satisfies the boundary conditions can be expressed as a linear combination of them. The true wavefunction vector $\mathbf{y}(x)$ can thus be expressed as

$$
\begin{aligned}
& \mathbf{y}(x)=\mathbf{Y}_{L}(x) \mathbf{c}_{L}, \quad x \leq x_{\text {match }} \\
& \mathbf{y}(x)=\mathbf{Y}_{R}(x) \mathbf{c}_{R}, \quad x \geq x_{\text {match }}
\end{aligned}
$$

where $\mathbf{Y}_{L}$ and $\mathbf{Y}_{R}$ are the wavefunction matrices propagated from $a$ and $b$ and $\mathbf{c}_{L}$ and $\mathbf{c}_{R}$ are $x$-independent column vectors that must be found. For an acceptable wavefunction, both $\mathbf{y}$ and its derivative must match at $x=x_{\text {match }}$,

$$
\begin{aligned}
\mathbf{y}\left(x_{\text {match }}\right) & =\mathbf{Y}_{L}\left(x_{\text {match }}\right) \mathbf{c}_{L}=\mathbf{Y}_{R}\left(x_{\text {match }}\right) \mathbf{c}_{R} \\
\mathbf{y}^{\prime}\left(x_{\text {match }}\right) & =\mathbf{Y}_{L}^{\prime}\left(x_{\text {match }}\right) \mathbf{c}_{L}=\mathbf{Y}_{R}^{\prime}\left(x_{\text {match }}\right) \mathbf{c}_{R} .
\end{aligned}
$$

These two equations can be combined into the single equation

$$
\left[\begin{array}{cc}
\mathbf{Y}_{L} & \mathbf{Y}_{R} \\
\mathbf{Y}_{L}^{\prime} & \mathbf{Y}_{R}^{\prime}
\end{array}\right]\left[\begin{array}{c}
\mathbf{c}_{L} \\
-\mathbf{c}_{R}
\end{array}\right]=\mathbf{0}
$$

where the matrix on the left-hand side is evaluated at $x=x_{\text {match. }}$. It is a matrix of order $2 n \times 2 n$ and is a function of the energy at which the wavefunctions are calculated. A non-trivial solution of Eq. (29) exists only if the determinant of the matrix on the left is zero, and this is only true if the energy used is an eigenvalue of the coupled equations. It is then straightforward to find the vectors $\mathbf{c}_{L}$ and $\mathbf{c}_{R}$. 
As for the one-dimensional case it is possible to construct a Newton-Raphson process to localise the eigenvalues by using the derivatives of the wavefunction with respect to $E$. As shown in [9] and [12 these derivatives can be propagated by the $\mathrm{CP}$ methods simultaneously with the wavefunction itself at a relatively low extra cost.

The procedure described above requires that the wavefunction matrix and its derivative be propagated explicitly. However there is one well known difficulty in the theory of close-coupled equations. The propagation of the wavefunction into the so-called classically forbidden region (where $V(x)>E$ ) is numerically unstable. It is due to the fact that the exponentially growing component $y_{j}$ of the wave function in the most strongly closed $\left(V_{j j}(x)>E\right)$ channel soon dominates the entire wave function matrix and destroys the required linear independence of the solutions. One approach to overcoming the difficulty is to apply certain stabilizing transformations during propagation (see [4]). In 9] a stabilizing transformation based on LU decomposition is described for the propagation by $\mathrm{CP}$ methods. After some propagation steps this regularization procedure can be applied to re-establish the linear independence of the columns.

Another way to avoid the difficulty is to use a so-called "invariant imbedding" method, in which the propagated quantity is not the wave function matrix $\mathbf{Y}(x)$ but rather its logarithmic derivative $\mathbf{Y}^{\prime}(x) \mathbf{Y}(x)^{-1}$ (see e.g. [10]). For the CP methods we can use the knowledge of the components of the matrix $\mathbf{T}^{f}$ and $\mathbf{T}^{b}$ for the propagation of the $\log$ derivative of the solution $\Psi=\mathbf{Y}^{\prime} \mathbf{Y}^{-1}$ :

$$
\begin{array}{r}
\boldsymbol{\Psi}(X+h)=\left[\mathbf{u}^{\prime}(h)+\mathbf{v}^{\prime}(h) \boldsymbol{\Psi}(X)\right][\mathbf{u}(h)+\mathbf{v}(h) \boldsymbol{\Psi}(X)]^{-1} \\
\boldsymbol{\Psi}(X)=\left[-\mathbf{u}^{\prime}(h)+\mathbf{u}(h) \boldsymbol{\Psi}(X+h)\right]\left[\mathbf{v}^{\prime}(h)-\mathbf{v}(h) \boldsymbol{\Psi}(X+h)\right]^{-1} .
\end{array}
$$

The matching condition can then also be expressed in terms of $\boldsymbol{\Psi}(x)$.

\section{Example}

As a test problem, we consider a multichannel Schrödinger problem of which the exact eigenvalues are known

$$
\mathbf{y}^{\prime \prime}=\left[\begin{array}{cc}
x^{2}-E & -1 \\
-1 & x^{2}-E
\end{array}\right] \mathbf{y}, \quad \mathbf{y}(0)=\mathbf{y}(10)=\mathbf{0} .
$$

The CPM $\{10,8\}$ method was used as the propagation method and at $x_{\text {match }}$ a Newton-Raphson process was applied. Doing all calculations in standard precision (16 significant figures), the results listed in Table 1 were generated. The table shows the first four eigenvalue estimations for different values of the input tolerance tol. The second column contains the number of meshpoints $m$ in the corresponding partition. For more details on the generation of the partition, we refer to [12].

Knowing that the first four exact eigenvalues are 2, 4, 6 and 8, it is clear that we were able to obtain very precise eigenvalue approximations. Also other (more complicated) testcases show that the multichannel $\operatorname{CPM}\{P, N\}$ methods have the power to estimate eigenvalues accurately. Moreover, the $\operatorname{CPM}\{P, N\}$ methods are very efficient to use in a shooting process: the relatively time consuming 
Table 1. Approximations of the first four Eigenvalues of Problem (31)

\begin{tabular}{llllll}
\hline$t o l$ & $m$ & $n=0$ & $n=1$ & $n=2$ & $n=3$ \\
\hline $10^{-6}$ & 23 & 1.99999995 & 3.999999956 & 5.99999991 & 7.99999991 \\
$10^{-8}$ & 38 & 1.9999999995 & 3.9999999995 & 5.9999999992 & 7.9999999992 \\
$10^{-10}$ & 62 & 1.999999999998 & 3.999999999998 & 5.999999999998 & 7.999999999998 \\
$10^{-12}$ & 102 & 1.99999999999998 & 3.99999999999998 & 5.99999999999999 & 7.99999999999999 \\
\hline
\end{tabular}

task of generating the partition can be performed only once (at the very beginning of the run) and can be completely separated from the repeatedly asked but fast executable task of propagating the solution at various values of $E$.

\section{References}

1. Allison, A.C.: The numerical solution of coupled differential equations arising from the Schrödinger equation. J. Comput. Phys. 6 (1970) 378-391

2. Allison, A.C.: The numerical solution of the equations of molecular-scattering. Adv. At. Mol. Phys. 25 (1988) 323-341

3. Cooley, J.W.: An Improved Eigenvalue Corrector Formula for Solving the Schrödinger Equation for Central Fields. Math. Comput. 15 (1961) 363-374

4. Gordon, R.G.: New Method for Constructing Wavefunctions for Bound States and Scattering. J. Chem. Phys. 51 (1969) 14-25

5. Hutson, J.M.: Coupled channel methods for solving the bound-state Schrödinger equation. Comput. Phys. Commun. 84 (1994) 1-18.

6. Ixaru, L.Gr.: Numerical Methods for Differential Equations and Applications. Reidel, Dordrecht-Boston-Lancaster (1984)

7. Ixaru, L.Gr., De Meyer, H., Vanden Berghe, G.: CP methods for the Schrödinger equation, revisited. J. Comput. Appl. Math. 88 (1997) 289-314

8. Ixaru, L.Gr., De Meyer, H., Vanden Berghe, G.: SLCPM12 - A program for solving regular Sturm-Liouville problems. Comp. Phys. Comm. 118 (1999) 259-277

9. Ixaru, L.Gr.: LILIX - A package for the solution of the coupled channel Schrödinger equation. Comput. Phys. Commun. 147 (2002) 834-852

10. Johnson, B.R.: Renormalized Numerov method applied to calculating bound-states of coupled-channel Schrödinger equation. J. Chem. Phys 69 (1978) 4678-4688

11. Ledoux, V., Van Daele, M., Vanden Berghe, G.: CP methods of higher order for Sturm-Liouville and Schrödinger equations. Comput. Phys. Commun. 162 (2004) 151-165

12. Ledoux, V., Van Daele, M., Vanden Berghe, G.: $\operatorname{CPM}\{P, N\}$ methods extended for the solution of coupled channel Schrödinger equations. Comput. Phys. Commun. 174 (2006) 357-370

13. Ledoux, V., Van Daele, M., Vanden Berghe, G.: MATSLISE: A MATLAB package for the Numerical Solution of Sturm-Liouville and Schrödinger equations. ACM Trans. Math. Softw. 31 (2005)

14. Levine, R.D.: Adiabatic approximation for nonreactive subexcitation molecular collisions. J. Chem. Phys. 49 (1968) 51

15. Rykaczewski, K., Batchelder, J.C., Bingham, C.R. et al.: Proton emitters ${ }^{140} \mathrm{Ho}$ and ${ }^{141}$ Ho: Probing the structure of unbound Nilsson orbitals. Phys. Rev. C. 60 (1999) 011301 